\title{
COLONIC CONTENT IN HEALTH AND ITS RELATION TO FUNCTIONAL GUT SYMPTOMS
}

\author{
Short title: colonic content
}

R. Alvaro Bendezú, M.D ${ }^{1}$ Elizabeth Barba, M.D. 1, Emanuel Burri, M.D. ${ }^{1,2}$, Daniel Cisternas ${ }^{1,3}$, Anna Accarino, M.D. ${ }^{1}$, Sergi Quiroga, M.D. ${ }^{4}$, Eva Monclus ${ }^{5}$, Isabel Navazo, M.D. ${ }^{5}$, Juan-R. Malagelada, M.D. ${ }^{1}$, and Fernando Azpiroz, M.D. 1

1. Digestive System Research Unit, University Hospital Vall d'Hebron; Centro de Investigación Biomédica en Red de Enfermedades Hepáticas y Digestivas (Ciberehd), Barcelona, Spain; Departament de Medicina, Universitat Autònoma de Barcelona, 08193 Bellaterra (Cerdanyola del Vallès), Spain.

2. Current address: Department of Internal Medicine, Stadspital Triemli, Zürich, Switzerland.

3. Current address: Clínica Alemana de Santiago, Facultad de Medicina Clínica Alemana, Universidad del Desarrollo, Santiago, Chile.

4. Radiology Department, University Hospital Vall d'Hebron, Barcelona, Spain

5. Departamento de Lenguajes y Sistemas Informáticos, Universitat Politécnica de Catalunya, Spain.

$\begin{array}{ll}\text { Abbreviations: } & \text { CT: computed tomography } \\ & \text { IBS: irritable bowel syndrome }\end{array}$

Corresponding author: $\quad$ Fernando Azpiroz, M.D.

Digestive System Research Unit

Hospital General Vall d'Hebron

08035-Barcelona, Spain

Phone: (34) 932746222

Fax: (34) 934894456

E-mail: azpiroz.fernando@gmail.com 


\begin{abstract}
Background. Gut content may be determinant in the generation of digestive symptoms, particularly in patients with impaired gut function and hypersensitivity. Since the relation of intraluminal gas to symptoms is only partial, we hypothesized that non-gaseous component may play a decisive role.

Methods. Abdominal CT scans were evaluated in healthy subjects during fasting and after a meal $(n=15)$ and in patients with functional gut disorders during basal conditions (when they were feeling well) and during an episode of abdominal distension $(n=15)$. Colonic content and distribution were measured by an original analysis program.
\end{abstract}

Key results. In healthy subjects both gaseous $(87 \pm 24 \mathrm{~mL})$ and non-gaseous colonic content $(714 \pm 34 \mathrm{~mL})$ were uniformly distributed along the colon. In the early postprandial period gas volume increased (by $46 \pm 23 \mathrm{~mL}$ ), but nongaseous content did not, although a partial caudad displacement from the descending to the pelvic colon was observed. No differences in colonic content were detected between patients and healthy subjects. Symptoms were associated with discrete increments in gas volume. However, no consistent differences in non-gaseous content were detected in patients between asymptomatic periods and during episodes of abdominal distension.

Conclusions \& Inferences. In patients with functional gut disorders, abdominal distension is not related to changes in non-gaseous colonic content. Hence, other factors, such as intestinal hypersensitivity and poor tolerance of small increases in luminal gas may be involved.

Key words: colonic content; intestinal gas; abdominal distension; functional gut disorders; food ingestion. 


\section{Key messages}

- Using an original program for abdominal CT image analysis our study describes the distribution of non-gaseous content in the undisturbed colon.

- Meal ingestion induced a caudad displacement of non-gaseous content in the distal colon.

- Patients with functional gut disorders complaining of episodic abdominal distension did not show differences in non-gaseous colonic content versus healthy subjects, either when asymptomatic or during distension episodes. 


\section{INTRODUCTION}

The mechanism of symptoms in functional gut disorders has not been fully elucidated. Two major pathophysiological mechanisms are currently accepted: abnormal motility and increased gut sensitivity (1;2). We hypothesized that in patients with functional bowel disorders even small variations in intraluminal content could trigger abnormal sensory-reflex responses and elicit symptoms. Among gut contents, and particularly in the colon, increased gas volume has been proposed as a symptom triggering culprit. During the past 15 years our laboratory has conducted a systematic research program quantitating normal intestinal gas handling, content and distribution, uncovering abnormalities in patients with functional gut disorders, particularly in patients complaining of abdominal bloating and distension, symptoms frequently attributed to intestinal gas.

Indeed, intestinal gas is easily visualized in abdominal CT scans. We developed and validated an original CT analysis program to measure the volume of gas in different regions of the gut (3-6). Using this program we described the pattern of intestinal gas in healthy subjects and in patients with episodic abdominal distension (7). During asymptomatic periods no differences were observed between both groups. During episodes of abdominal distension some patients exhibited an intraluminal gas pattern outside the normal range, but the abnormalities were relatively mild. Furthermore, in most patients no abnormalities in intraluminal gas volume could be detected during the symptomatic episodes. 
Expanding our initial hypothesis, we further postulated that gas may not be the sole offending component, and that the solid-liquid component of gut contents may play a role distending certain segments of the lower gut. At this stage, we developed a program for analysis of the non-gaseous (solid-liquid) content of the gut and applied it to measure colonic content. With this new method we revised the CT scans previously acquired (7) with two aims, first, to describe the basal pattern of colonic content in healthy subjects and the physiological variations related to eating, and second, to describe the pattern of content in patients with functional gut symptoms and to determine the temporal relation of contents to symptoms. To these goals we analyzed a series of paired studies in healthy subjects during fasting and after a meal, and in a group of patients with episodic abdominal distension during symptomatic episodes and during asymptomatic intervals between distension episodes.

\section{MATERIAL AND METHODS}

\section{Participants}

Healthy individuals [11 women, 4 men; $39 \pm 4$ years age (mean $\pm S E$ ); 19 62 years range] with no gastrointestinal complaints and patients complaining of episodic abdominal distension i.e. severe distension episodes in contrast to mild or no distension in-between episodes ( 15 women; $53 \pm 3$ years age; $37-74$ years age), were included in the study. Patients were recruited from the Gastroenterology outpatient clinic and healthy subjects by public advertisement (3;7). All participants were instructed to fill-out a digestive symptoms questionnaire based on Rome criteria; this information was used to establish a diagnosis in patients, and to verify the absence of symptoms in healthy 
subjects. All patients $(n=15)$ were diagnosed as having functional disorders based on Rome III criteria (8), 7 of them as functional bloating (8.3 \pm 2.2 bowel movement/wk, $3.3 \pm 0.4$ score on the Bristol stool form scale), 4 constipationpredominant IBS (1.3 \pm 0.3 bowel movements/wk, 2.1 \pm 0.3 Bristol score), 3 with alternating IBS $(2.1 \pm 0.1$ bowel movements/wk, $1.3 \pm 0.1$ Bristol score $)$, and 1 diarrhea-predominant IBS. The protocols of the specific studies had been approved by the Institutional Review Board of the University Hospital Vall d'Hebron and all subjects gave their written informed consent to participate in the studies.

\section{CT scanning}

Abdominal CT scans were obtained with a helical multislice CT scanner, exposure $120 \mathrm{kV}$ and $50 \mathrm{mAs}$, using the available dose reduction options (tube current modulation). Section thickness and image reconstruction depended on the scanner model (2.5-mm thickness, 1.6-mm interval reconstruction with Mx8000, Philips Medical Systems, Best, The Netherlands; 2-mm thickness, 1.5-mm interval reconstruction with Somatom Sensation 16 or Somatom Definition AS, Siemens Medical Solutions, Forchheim, Germany). With these characteristics the total effective dose was $2.4 \mathrm{mSv}$, similar to the dose of a CT colography and approximately $1 / 4$ of the dose of a standard CT scan. Images were obtained in the supine position during a single breath-hold. No oral or intravenous contrast medium was administered. At the time of each CT scan, the participant's subjective sensation of abdominal distension was measured on a graphic rating scale graded from 0 (no distension) to 6 (extremely severe distension). 


\section{Experimental design}

All participants underwent two abdominal CT scans. Healthy subjects were studied: a) in the morning during fasting and b) in the postprandial period (109 $\pm 21 \mathrm{~min}$ after a meal). Patients with episodic abdominal distension were studied on two different symptomatic status: a) during a spontaneous episode of severe distension, and b) at baseline (in between episodes) when they considered their abdomen showed minimal or no distension. All healthy subjects included in a previous study with paired fasting and postprandial scans were selected for the current study (3); patients were randomly selected among those with paired basal and distension scans included in previous studies (7). CT scanning and analyses were performed blindly, with the investigator unaware of the condition (patient or healthy subject) and the symptom status (basal or symptomatic) of the participants.

\section{Data analysis}

Morphovolumetric analysis of CT images was performed using an original software program specifically developed in our laboratory and. Total colonic content was measured using a computer-assisted semiautomatic method: the operator selects in each image the intracolonic content, which is then measured by anisotropic contrast enhancement and region growing algorithms. Gaseous component of intracolonic content was automatically measured by applying a user-defined threshold $(3 ; 4)$. Using a three-dimensional reconstruction program with $360^{\circ}$ rotation over the three dimensions, volumes in 
selected regions of the colon were also measured. Analysis of each scan involves about 60 min operator's time. The reproducibility of the measurements was originally validated in vivo by measuring intestinal gas before and after injection of known volumes of air within the gut (15). The software used in this study was built with two open-source toolkits: the Insight Segmentation and Registration toolkit (9) and the Visualization toolkit, both used for 3D computer graphics, image processing and visualization (10).

\section{Statistical analysis}

Statistical analysis was performed using the SPSS 18.0 statistical package. Mean \pm SE of the features measured were calculated in each group of participant. The Kolmogorov-Smirnov test was used to check the normality of data distribution. Parametric normally-distributed data were compared by Student's $t$-test for paired or unpaired data; otherwise, the Wilcoxon signed rank test was used for paired data and the Mann-Whitney $U$ test for unpaired data.

\section{RESULTS}

\section{Healthy subjects}

Total colonic content during fasting was $801 \pm 45 \mathrm{~mL}$ of which $87 \pm 24 \mathrm{~mL}$ were gas and $714 \pm 34 \mathrm{~mL}$ non-gaseous (solid/liquid) content (Figure 1 and 2). Both gas and non-gaseous content were uniformly distributed along the colon (Figure 3). The reproducibility of the measurements during fasting and after the meal was good; in the early postprandial period, total colonic volume increased slightly, but even small changes in the distribution of both gaseous and nongaseous components were consistently noted (Figures 2 and 3): the non- 
gaseous content decreased in the descending colon (by $15 \pm 8 \mathrm{~mL} ; \mathrm{p}=0.033$ ) and increased proportionally in the pelvic colon (by $19 \pm 10 \mathrm{~mL} ; \mathrm{p}=0.040$ ), whereas gas content increased selectively in the proximal part (ascending; $p=0.036$ ) and distal part of the colon (descending and pelvic; $p \leq 0.034$ for both).

\section{Patients}

Patients studied during basal conditions, in-between distension episodes, reported mild symptoms (2.2 \pm 0.3 distension score) and no consistent differences in colonic content gaseous and non-gaseous, were observed as compared to healthy subjects (Figures 2 and 3 ). The same patients during episodes of abdominal distension (4.6 \pm 0.4 . distension score) showed a mild increase in colonic gas content (up to $128 \pm 32 \mathrm{~mL}$ from $94 \pm 3 \mathrm{~mL}$ in the basal scan; $p=0.059$ ), but no significant differences in the non-gaseous content of the colon were observed); the reproducibility of the measurements during basal conditions and episodes of distension was good (Figure 2). The severity of symptoms during distension episodes (either absolute values or increase from basal) did not correlate with changes in intracolonic fecal mass. 


\section{DISCUSSION}

Our study describes and quantitates the normal pattern of content in the undisturbed colon, during fasting and the early postprandial period. The study further shows that patients with functional gut symptoms do not exhibit detectable abnormalities in non-gaseous colonic content.

The volume of colonic content measured in the present study by CT is remarkably similar to the volumes measured by MRI both by us and others (1113). The volume of colonic content is much smaller than previously expected, and considering that the daily fecal output is around $150 \mathrm{~mL}$, the turn-over of colonic biomass seems particularly rapid (14). Interestingly, intraindividual differences in non-gaseous colonic content are relatively small; the volume of gas is much more variable, but this variability involves relatively small quantities of intracolonic gas, because differences in absolute gas volumes among individuals are not large. To note, the reproducibility of the measurements was good and the technique allowed detection of relatively small, but consistent and statistically significant intraindividual variations.

The distribution of non-gaseous content within the undisturbed colon is quite uniform, indicating that the metabolic activity of colonic biomass takes place all along the entire length of the colon. Meal ingestion was not associated with changes in colonic content in the early postprandial period, probably because the postprandial scan was obtained before the bulk of unabsorbed meal residues arrived from the small bowel. However, meal ingestion induced a redistribution of non-gaseous content that decreased in the descending colon and proportionally increased in the pelvic segments. Conceivably, this caudad displacement of biomass is operated by the gastro-colonic reflex activated by 
ingestion of the meal $(15 ; 16)$. The situation may change at a later stage of the postprandial period when unabsorbed residues of small bowel chime enter the colon. Thus, in a recent study we observed a consistent increment in content all along the colon $6 \mathrm{~h}$ after ingestion of a test meal rich in residues (17).

The gas component of intracolonic content after the meal changes according to a different pattern than the non-gaseous component. As previously described $(3 ; 7)$, the absolute volume of colonic gas increased during the early postprandial period. Gas increased in the ascending colon, which could be due to emptying of gas from the small bowel by a gastro-ileal reflex, because fermentable residues had probably not arrived at the time of scanning; at the same time an increase was observed in the distal colon, conceivably due to a caudad movement driven by the gastro-colonic reflex.

In patients complaining of abdominal distension, changes in gas content and distribution have been previously reported (7). In that earlier study a relatively large population of patients was studied, allowing application of sophisticated analysis by machine learning techniques. This analysis showed that in-between symptomatic episodes, the intraluminal gas pattern in patients was undistinguishable from healthy subjects. During symptomatic episodes, a substantial proportion of patients were classified outside the normal range by a combination of abnormalities, although no single characteristic was found discriminant. The present data in a subpopulation of these patients corroborates the increase in gas content associated to abdominal distension, although the differences failed to reach statistical significance due to the smaller sample size. We acknowledge that healthy subjects and patients were not matched, but previous studies did not detect differences related to sex, age or BMI (3-7). 
The original contribution of the present study is that, contrary to our hypothesis, no abnormalities in the non-gaseous component of colonic content were observed in patients regardless of whether they were asymptomatic or during one of their usual episodes of abdominal distension. Indeed, the range of individual values in both populations overlap (see Figure 2), suggesting that the lack of difference was not related to the sample size.

These data indicate that abdominal distension in these patients may develop with a normal amount of solid/liquid mass inside their colons. Consequently, their symptoms are more likely related to luminal gas, which makes sense, considering the temporal variations both of symptoms and gas content, as opposed to the stability of non-gaseous colonic content. Furthermore, increased colonic gas during symptomatic episodes is quantitatively modest and only detected in a proportion of symptomatic patients; hence, other factors, such as intestinal hypersensitivity, may be involved. Content in other segment of the gut, such as the stomach of small bowel, may also play a role. Futures studies on gut content in relation to symptoms, requiring repeated imaging over time, may benefit from less invasive technologies that do not involve radiation, such as MRI (11-13). 


\section{Acknowledgments}

The authors thank Anna Aparici, and Purificación Rodriguez for technical support and Gloria Santaliestra for secretarial assistance.

\section{Funding}

This work was supported in part by the Spanish Ministry of Economy and Competitiveness (Dirección General de Investigación Científica y Técnica, SAF 2013-43677-R); Ciberehd is funded by the Instituto de Salud Carlos III. Dr. Burri was supported by grants from the Freiwillige Akademische Gesellschaft (Basel, Switzerland) and the Gottfried und Julia Bangerter-Rhyner-Stiftung (Bern, Switzerland).

\section{Author Contributions:}

AB. Data analysis

EBarba. Study management and conduction of experiments.

EBurri. Study management and conduction of experiments.

DC. Conduction of experiments.

AA. Study design, supervision of studies, data analysis and manuscript revision. $\mathrm{SQ}$. Analysis of CT images.

EM. Adaptation of CT analysis program.

IN. Adaptation of CT analysis program.

J-RM. Data interpretation, manuscript revision.

FA. Study design, data interpretation, and manuscript preparation.

\section{No competing interests declared}




\section{References}

(1) Kellow JE, Azpiroz F, Delvaux M, Gebhart GF, Mertz H, Quigley EM, et al. Applied principles of neurogastroenterology: physiology/motility sensation. Gastroenterology 2006;130:1412-20.

(2) Azpiroz F, Bouin M, Camilleri M, Mayer EA, Poitras P, Serra J, et al. Mechanisms of hypersensitivity in IBS and functional disorders. Neurogastroenterol Mot 2007;19:62-88.

(3) Perez F, Accarino A, Azpiroz F, Malagelada J-R. Gas distribution within the human gut: effect of meals. Am J Gastroenterol 2007;102:842-9.

(4) Accarino A, Perez F, Azpiroz F, Quiroga S, Malagelada JR. Abdominal distension results from caudo-ventral redistribution of contents. Gastroenterology 2009;136:1544-51.

(5) Accarino A, Perez F, Azpiroz F, Quiroga S, Malagelada J-R. Intestinal gas and bloating: effect of prokinetic stimulation. Am J Gastroenterol 2008;103:2036-42.

(6) Barba E, Burri E, Accarino A, Cisternas D, Quiroga S, Monclus E, et al. Abdomino-thoracic mechanisms of functional abdominal distension and correction by biofeedback. Gastroenterology 2015;148(4):732-8.

(7) Bendezú A, Barba E, Burri E, Cisternas D, Malagelada C, Segui S, et al. Intestinal gas content and distribution in health and in patients with functional gut symptoms. Neurogastroenterol Motil 2015;27(9):1249-57.

(8) Thompson WG, Longstreth G, Drossman DA, Heaton K, Irvine EJ, Muller-Lissner S. Functional bowel disorders and Functional abdominal pain. In: Drossman DA, Corazziari E, Talley NJ, Thompson WG, Whitehead WE, editors. The Functional Gastrointestinal Disorders. 2nd ed. Mc Lean, VA: Degnon Associates; 2000. p. 351-432.

(9) Ibañez L, Schroeder W, Ng L, Cates J. The ITK Software Guide. Kitware Inc 2003.

(10) Schroeder W, Martin K, Lorensen B. The visualization toolkit, an object oriented approach to 3D graphics. Kitware Inc 2002;3rd edition.

(11) Bendezú A, Merino X, Monclus E, Izquierdo M, Accarino A, Navazo I, et al. El cólon como órgano metabólico: medición del contenido. Rev Esp Enf Digest 2014;106(I):A172.

(12) Bendezú A, Accarino A, Quiroga S, Merino X, Izquierdo M, Monclus E, et al. Efecto de la ingesta sobre el contenido del tubo digestivo en condiciones fisiológicas. Rev Esp Enf Digest 2015;107(I):A168. 
(13) Pritchard SE, Marciani L, Garsed KC, Hoad CL, Thongborisute W, Roberts $\mathrm{E}$, et al. Fasting and postprandial volumes of the undisturbed colon: normal values and changes in diarrhea-predominant irritable bowel syndrome measured using serial MRI. Neurogastroenterol Motil 2014 Jan;26(1):124-30.

(14) Mego M, Mendez S, Accarino A, Azpiroz F. Efecto acumulativo de las comidas en el metabolismo de la microbiota y la producción de gas intestinal. Rev Esp Enf Digest 2015;107(I):A167.

(15) Simren M, Ringstrom G, Bjornsson ES, Abrahamsson H. Treatment with hypnotherapy reduces the sensory and motor component of the gastrocolonic response in irritable bowel syndrome. Psychosom Med 2004 Mar;66(2):233-8.

(16) Suttor VP, Ng C, Rutkowski S, Hansen RD, Kellow JE, Malcolm A. Colorectal responses to distension and feeding in patients with spinal cord injury. Am J Physiol Gastrointest Liver Physiol 2009 Jun;296(6):G1344-G1349.

(17) Bendezú A, Mego M, Merino X, Accarino A, Monclus E, Izquierdo M, et al. Colonic biomass: effect of diet, meals and defecation. United European Gastroenterology Journal 2015;3(5S):A505. 


\section{FIGURE LEGENDS}

Figure 1. Independent representation of gaseous and non-gaseous components of colonic content from a CT scan in a healthy subject.

Figure 2. Colonic content. Total, non-gaseous and gaseous content are represented. Data are individual values (and means) in healthy subjects during fasting and in the early postprandial period, and in patients during basal conditions and during an episode of abdominal distension.

Figure 3. Distribution of colonic content. Figure shows total, non-gaseous and gaseous content in different colonic segments: ascending (A), transverse $(\mathbf{T})$, descending (D) and pelvic (P). Data are mean values $\pm S E$ in healthy subjects (fast vs early postprandial period) and in patients (basal conditions vs episodes of abdominal distension). No consistent difference between patients and healthy subjects were detected. 
Figure 1
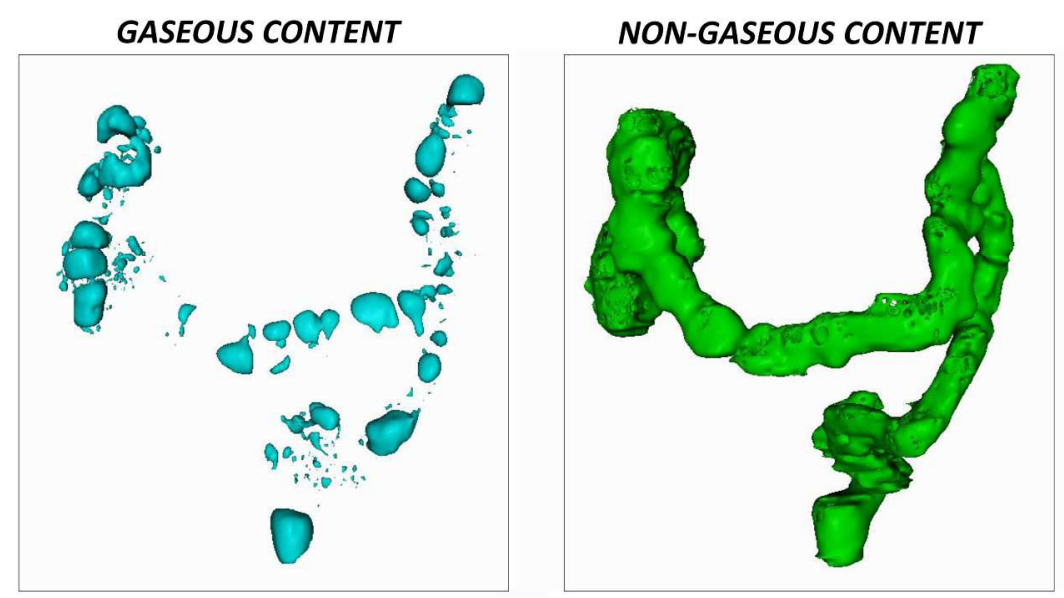
Figure 2

Colonic content. Total, non-gaseous and gaseous content are represented. Data are individual values (and means) in healthy subjects during fasting and in the early postprandial period, and in patients during basal conditions and during an episode of abdominal distension. $190 \times 254 \mathrm{~mm}(300 \times 300 \mathrm{DPI})$ 
Figure 3

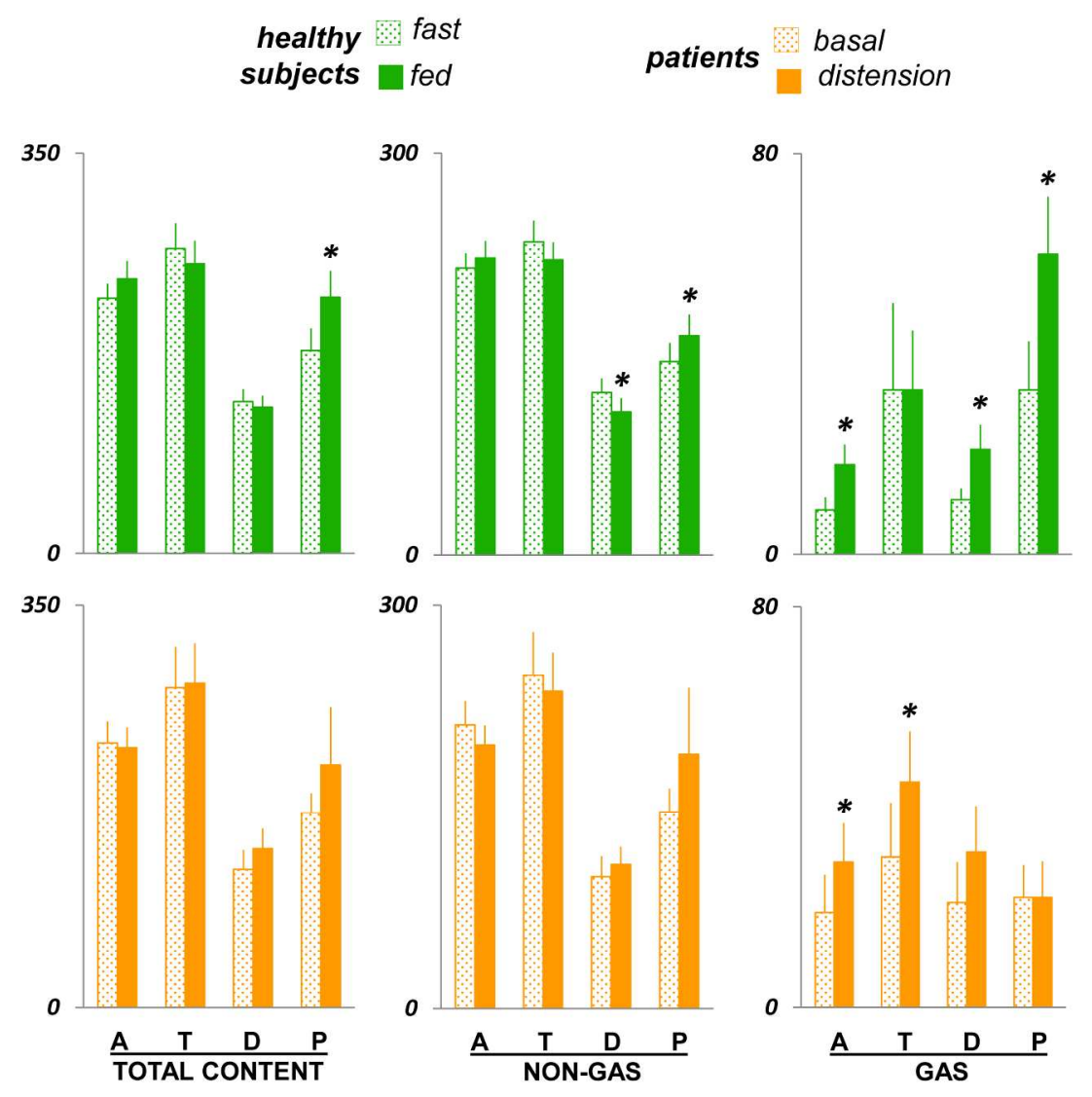

\footnotetext{
Distribution of colonic content. Figure shows total, non-gaseous and gaseous content in different colonic segments: ascending (A), transverse $(T)$, descending $(D)$ and pelvic $(P)$. Data are mean values $\pm S E$ in healthy subjects (fast vs early postprandial period) and in patients (basal conditions vs episodes of abdominal distension). No consistent difference between patients and healthy subjects were detected. $190 \times 254 \mathrm{~mm}(300 \times 300 \mathrm{DPI})$
} 